\title{
A Multi-Agent Platform for Hospital Interoperability
}

\author{
Luciana Cardoso $^{1}$, Fernando Marins ${ }^{1}$, Filipe Portela ${ }^{2}$, Manuel Santos ${ }^{2}$, \\ António Abelha ${ }^{1}$, and José Machado ${ }^{1}$ * \\ ${ }^{1}$ University of Minho, Computer Science and Technology Centre, Braga, Portugal, \\ ${ }^{2}$ University of Minho, Algoritmi research Centre, Guimarães, Portugal \\ \{a55524, a55561\}@alunos.uminho.pt \\ \{cfp,mfs\}@dsi.uminho.pt \\ \{abelha, jmac\}@di.uminho.pt
}

\begin{abstract}
The interoperability among the Health Information Systems is a natural demand nowadays. The Agency for Integration, Diffusion and Archive of Medical Information (AIDA) is a Multi-Agent System (MAS) specifically developed to guarantee interoperability in health organizations.

This paper presents the Biomedical Multi-agent Platform for Interoperability (BMaPI) integrated in AIDA and it is used by all hospital services which communicates with AIDA, one of the examples is the Intensive Care Unit. The BMaPI main objective is to facilitate the communication among the agents of a MAS. It also assists the interaction between humans and agents through an interface that allows the administrators to create new agents easily and to monitor their activities in real time. Due to the BMaPI characteristics it is possible ensure the continuous work of the AIDA agents associated to INTCare system.

The BMaPI was installed in Centro Hospitalar do Porto successfully, increasing the functionality and overall usability of AIDA platform.
\end{abstract}

Keywords: Hospital Interoperability; Multi-Agent Systems; Agents Monitoring; AIDA; INTCare.

\section{Introduction}

Implementing technology in health organizations is increasing exponentially. The Health Information Systems (HIS) are one of the biggest examples of these technologies, which have played a key role in the workflow in these organizations nowadays. However, these systems are distributed and heterogeneous. The interaction among these systems is a crucial demand these days. In this way, the interoperability among the HIS becomes an indispensable feature in health organizations $[1,2]$.

Interoperability is the capacity of two systems interact between them, ensuring the understanding of the process and data exchanged on both sides [3].

\footnotetext{
* Corresponding Author
} 
Standards such as the Health Level Seven (HL7) avoid different structures of the transferred information and in this way the correct interpretation and communication are achieved among the HIS [4]. There are various technologies that have the ability to implement the interoperability among these systems, such as the Service Oriented Architecture (SOA), webservice interfaces, eXtended Markup Language (XML) and Multi-Agent Systems (MAS) [5]. This last one has demonstrated to be a powerful technology in the area of interoperability, addressing heterogeneous and distributed limitations $[1,5,6]$.

The Agency for Integration, Diffusion and Archive of Medical Information (AIDA) is an agent-based platform with the purpose of ensuring the interoperability among HIS [6]. INTCare is an Intelligent Decision Support System to Intensive Care Units which is also based on a MAS and it uses some data provided by AIDA platform to construct their models [7]. The correct working of AIDA agents it is fundamental to the success of INTCare system. However it fails in controlling and monitoring its own agents.

In this context, it emerges the Biomedical Multi-agent Platform for Interoperability (BMaPI). It was integrated into the AIDA platform and it enables the AIDA administrators to verify the agents functioning or to detect eventual failures in their performance in real time. Through the BMaPI, the administrators can manage successfully all the AIDA agents, knowing with the detail when a specific agent performs its activities. So, the administrators are able to select the most opportune period to execute changes, updates, maintenance and other operations, improving the AIDA performance.

This paper is divided into five sections. This first section introduces the work presented in this paper and the contextualization is also presented. The second section is a background that aims the importance of agents and MAS for the interoperability and it also presents the AIDA platform. Section 3 presents the archetype for monitoring the AIDA agents: the BMaPI, more specifically its features, its architecture and the description of its components. The results obtained after BMaPI implementation are demonstrated and discussed in Section 4. The last section exposes the major conclusions and the future work.

\section{Background}

\subsection{Multi-Agent Systems for Interoperability}

The multi-agent technology has excelled in the interoperability implementation in the HIS $[1,6,7]$. This technology is rather close to the concepts that characterize a distributed architecture. The success of the agent-based computing has strongly appeared as a result of its ability to work out problems and to make a new revolution in the development and analysis of software [6].

Intelligent agents are computational artifacts that are endowed with some proprieties such as the autonomy, reactivity, pro-activity and social skills. The autonomy and pro-activity are features that allow to plan and to perform tasks designed to achieve the proposed objectives, without direct human intervention. 
The reactivity enables the agents act according to the environment where they are inserted. Through their social skills, the agents interact with each other in order to obtain a common purpose. With these properties, the MAS demonstrate to be a strong technology for the interoperability implementation among the HIS $[1,6,7]$.

\section{$2.2 \quad$ AIDA}

Techniques based on Artificial Intelligence (AI) have shown great potential when introduced into the hospital environment. Most of these systems are focused in the area of systems integration and decision support systems [8]. So, the Agency for Integration, Diffusion and Archive of Medical Information (AIDA) is a solution developed by a research group of AI at the University of Minho. AIDA is already implemented in several portuguese health organizations, including the Centro Hospitalar do Porto (CHP). It is based on the agent-oriented paradigm and its MAS increases according to the necessity of each institution. This platform has shown great adaptability, modularity and effectiveness in the health organizations which is implemented. AIDA was created with the intent to aid medical applications and to manage the information flow through processing systems with an adaptable level of autonomy. The main objective is to make the HIS interoperable and to provide complementary tactics of diagnostics and therapeutics, through the diffusion and the integration of the information produced in a health organization. The agents are the basic unit of AIDA and they ensure the communication among heterogeneous systems, sending, receiving, managing and storing information and responding to requests timely and correctly $[5,6]$.

INTCare is an Intelligent Decision Support System optimized to Intensive Care Units and it is associated to AIDA. The main objective is provide new knowledge to the decision process automatically and in real-time [8]. INTCare platform uses intelligent agents [7] to support their process and automate some tasks. In this context, the BMaPI became an important platform not only to AIDA, but also to INTCare because it allows to monitor the agents which are directly associated to the system.

\section{Archetype for Agents Monitoring}

The Biomedical Multi-agent Platform for Interoperability was designed to allow MAS administrators to control and to manage a community of agents, allowing their survival and the interoperability in a heterogeneous environment. It is a platform that integrates all agents belonging to the MAS, regardless the machine where they perform their tasks. This is intended to ensure not only that all agents communicate each other, but also to create an interoperable environment. The communication among the agents are made through messages that meet certain structural rules defined by the FIPA (Foundation for Intelligent Physical Agents) called ACL (Agent Communication Language) [9]. 
According to the needs described by administrators of the AIDA platform, it was developed the BMaPI which aims: Ensure a greater control over the agents that constitute the AIDA; Facilitate the user's work in the creation and registration of new agents locally or remotely; Allow the user to enable and to disable services at the health unit, through the launch or stop of a particular agent; Facilitate the scheduling and rescheduling of the agents activity; and Monitor dynamically and in real time the agents activity.

\subsection{Architecture and Components Description}

The BMaPI was constructed respecting the specifications of a client/server architecture, where the server is able to communicate with multiple clients simultaneously dedicating a thread to each of them. The ACL messages are exchanged between the agents through sockets, which use TCP/IP protocols to ensure that information is transferred from an agent to another keeping the integrity of transferred data.

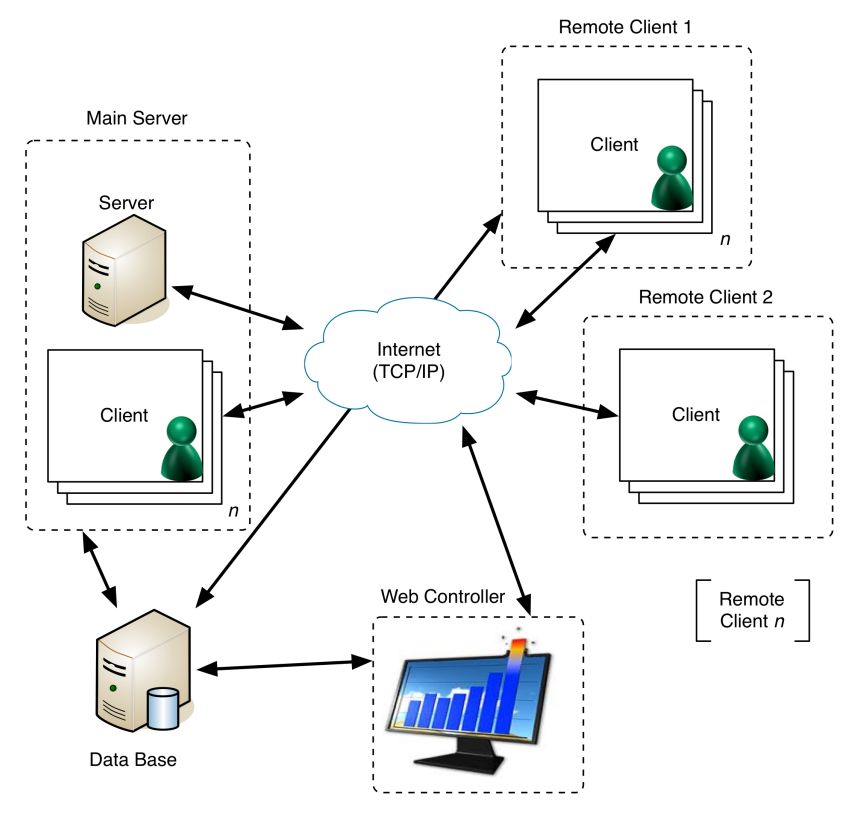

Fig. 1. Architecture of BMaPI.

Analyzing the BMaPI architecture presented in the Figure 1, is possible verify that it is composed of three distinct components: the Main Server, the Remote Clients and the Web Controller. The first two components are similar, the first is a server for the entire system and the second is the main client, which is installed on each machine and its purpose is to connect to the Main Server. The 
Web Controller is a user interface to control the agents, which allows the user to schedule the activities of each agent and also to monitor their activities.

\section{Main Server and Remote Client}

The Main Server is the only mandatory component, it makes the automatic boot (Figure 2) of the whole platform and it can be executed only once. The machine where this component runs is the main machine because it serves as the server but it can also host agents. The Remote Clients can be executed simultaneously on multiple machines, in all those that host agents. The main difference between these two components is that the Main Server might work remotely, i.e it has the ability to create and to send the agents to perform their activity in other machines.

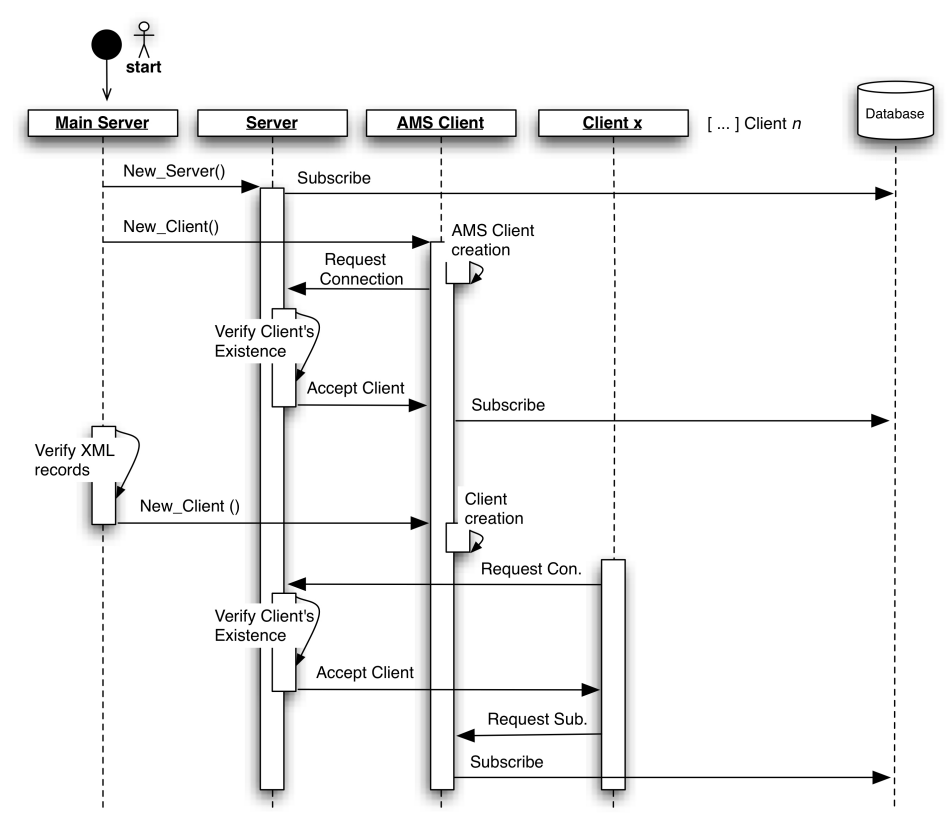

Fig. 2. Automatic boot process of BMaPI.

Analyzing the Figure 2 that represents the initialization of the BMaPI, it is possible to verify that the server creation is the first step followed by a record in the database of the IP and the port where it was created. After this the server is waiting for new connections from new clients. The first client automatically created and connected to the server is the Agent Management System (AMS). It is a special agent that exists in each machine and it is responsible for controlling all other agents hosted on this machine. After the AMS acceptance by the server, to finalize the creation process, some AMS information is stored in the database, 
namely its name, its state (active or inactive) and the machine IP wherein it operates. So that the administrator does not have to integrate all the agents in $\mathrm{BMaPI}$ each time there is a startup (of the machine or of the BMaPI), the agent information is stored locally in an XML file. This file serves so that whenever the platform is initialized all agents are created automatically.

The Remote Clients are started like the Main Server with the exception of the creation of the server. In these cases the request connection by the agents is sent to the server situated in the main machine where is the Main Server component.

\section{Web Controller}

The Web Controller constitutes the interface with the humans and it provides an attractive environment to control and to monitor the agents subscribed on the BMaPI. Exploiting each agent, it is possible access three pages: to visualize its properties; to schedule its activities; and to monitor its performed activities in real time and in a dynamic way. The properties page enables not only to analyze the properties of the agents, but also a summary of all activities performed by the agent since its creation until the present moment. More precisely: the date of the last activity; the duration of the last activity; the number of times that the agent was executed; the average duration of all activities of the agent; and the number of errors that occurred. The scheduling page gives the administrator a set of possibilities to program the activity of each agent. And the monitoring page has two kinds of dynamical graphs that correspond to the number of times that a specific agent performed its tasks and the average duration of the activities of the agent.

\section{Implementation Results}

In order to test the BMaPI functionalities, it was implemented on the CHP a hospital in the North of Portugal. A set of AIDA agents was integrated in BMaPI and their activities were controlled. The results presented in this paper are related to the period between 10 and 16 of September 2013 and to the agent 609. This agent called 609 is responsible to ensure the interoperability with the support system to the practices of nursing and its results are selected to be presented due to it be an agent that generally has some problems in its activities.

These results (Figure 3) represent the average duration of the activity of the agent 609 in seconds clustered per hour on September 11, 2013. From the graph presented in the Figure 3, it is concluded that the agent took less time to execute its tasks between 7AM and 8AM, with an average of 281.83 seconds and the maximum value was detected between $11 \mathrm{AM}$ and $12 \mathrm{PM}$, with a duration average of 457.62 seconds. It is possible to analyze that there is an abrupt growth between $8 \mathrm{AM}$ and $12 \mathrm{PM}$ followed by a gradual decrease until 11PM. This trend of the graph line can be related to the amount of data recorded in the nursing information system and consequently with the influx of patients to the nursing 


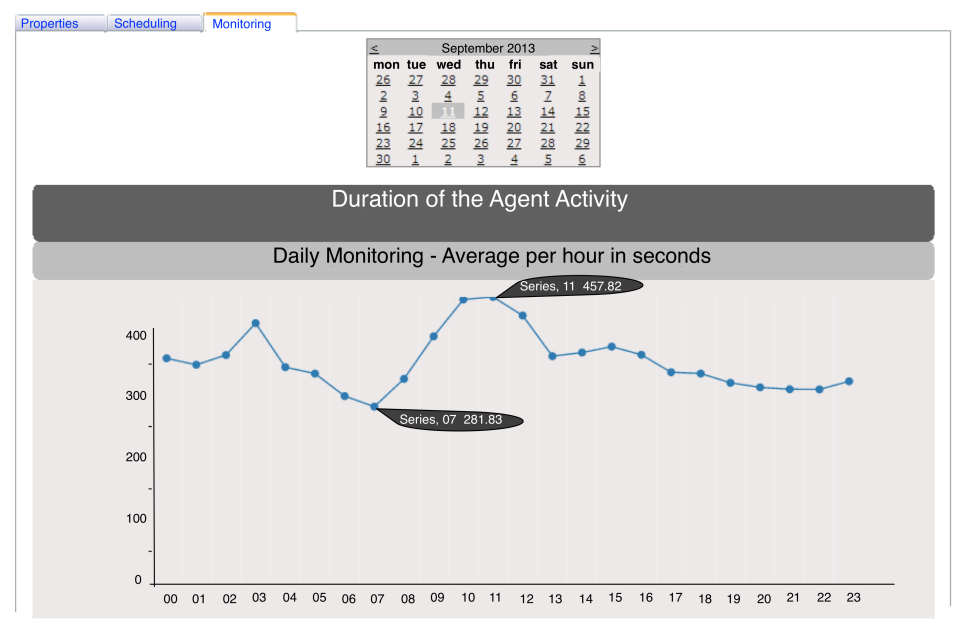

Fig. 3. Daily analysis of the duration of the activity of the agent 609 on September $11,2013$.

service. With all information that the web controller provides the administrators of the AIDA or of another MAS wherein the BMaPI was implemented can make a better manage of overall system and its subsystems (agents). They can know the period when the agents take more time to perform their activities and consequently decide which will be the best time to make routine changes to system upgrades, and other operations to improve overall system performance. Therefore the BMaPI when applied in a MAS improves its functionality, once it promotes functions that satisfy the users' needs. And it also offers more usability to the MAS, since being a system of easy understanding and operation with an attractive interface. With the BMaPI the interoperability is assured because the agents are able to communicate with each other in order to achieve mutual goals.

\section{Conclusions and Future Work}

It is possible concluded that the BMaPI implementation in the CHP offers to its AIDA and INTCare System a greater control of their agents, improving the functionality and the usability of both systems. INTCare is one of the system which it was directly benefited with the new features of AIDA. The BMaPI components developed with the agent-oriented programming revealed to be a powerful tool to control minutely these agents and to avoid possible failures and consequently, to improve the quality of the services delivered and the interoperability among the HIS. Besides that, a specific MAS (for example the AIDA or the INTCare) administrators are able to know the best period to apply operations like maintenance, updates or other changes, minimizing the disturbances in the MAS workflow. 
In the future, a communicative link can be established between BMaPI and a system developed by our research group, which enables the monitoring and the prevention of faults from the AIDA agents and machines [10]. This communication will enable to balance resources of AIDA (or other MAS) through the migration of its agents.

\section{Acknowledgement}

This work is financed with the support of the Portuguese Foundation for Science and Technology (FCT), with the grant SFRH/BD/70549/2010 and within project PEst-OE/EEI/UI0752/2014 and the contract PTDC/EEI-SII/1302/2012 - INTCare II.

\section{References}

1. Palazzo, L., Rossi, M., Dragoni, A.F., Claudi, A., Dolcini, G., Sernani, P.: A Multi-Agent Architecture for Health Information Systems. In: Advanced Methods and Technologies for Agent and Multi-Agent Systems. Volume 253 of Frontiers in Artificial Intelligence and Applications. IOS Press Ebooks (2013) 375-384

2. Weber-Jahnke, J., Peyton, L., Topaloglou, T.: eHealth System Interoperability. Information Systems Frontiers 14(1) (2012) 1-3

3. Oliveira, T., Novais, P., Neves, J.: Guideline formalization and knowledge representation for clinical decision support. Advances in Distributed Computing and Artificial Intelligence Journal (ADCAIJ 1(2) (2012) 1-12

4. Khan, W.A., Hussain, M., Latif, K., Afzal, M., Ahmad, F., Lee, S.: Process interoperability in healthcare systems with dynamic semantic web services. Computing 95(9) (2013) 837-862

5. Miranda, M., Duarte, J., Abelha, A., Machado, J., Neves, J.: Interoperability in Healthcare. In: Proceedings of the 24th European Simulation and Modelling Conference (ESM), Hasselt, Belgium, EUROSIS (2010)

6. Peixoto, H., Santos, M., Abelha, A., Machado, J.: Intelligence in Interoperability with AIDA. In: 20th International Symposium on Methodologies for Intelligent Systems. Volume 7661., World Intelligence Congress, Macau, Lecture Notes in Computer Science - Springer (2012)

7. Santos, M.F., Portela, F., Vilas-Boas, M.: Intcare: multi-agent approach for realtime intelligent decision support in intensive medicine. In: Proceedings of the International Conference on Agents and Artificial Intelligence (ICAART 2011), SciTePress (2011) 364-369

8. Portela, F., Santos, M.F., Machado, J., Abelha, A., Silva, Á.: Pervasive and Intelligent Decision Support in Critical Health Care Using Ensembles. In: Information Technology in Bio- and Medical Informatics. Volume 8060 of Lecture Notes in Computer Science (LNCS). Springer (2013) 1-16

9. Foundation for Intelligent Physical Agents (FIPA): FIPA ACL Message Structure Specification, http://www.fipa.org/specs/fipa00061/SC00061G.pdf (accessed on 22 February 2014). (2002)

10. Marins, F., Cardoso, L., Portela, F., Santos, M., Abelha, A., Machado, J.: Improving High Availability and Reliability of Health Interoperability Systems. In: Advances in Intelligent Systems and Computing. Springer (2014) 